\title{
Cerebrospinal Fluid Phosphate in Delirium after Hip Fracture
}

\author{
Ane-Victoria Idland ${ }^{\mathrm{a}, \mathrm{b}}$ Torgeir Bruun Wyller ${ }^{\mathrm{a}, \mathrm{c}}$ Randi Støen ${ }^{\mathrm{d}}$ \\ Gry Torsæter Dahle Frede Frihagen ${ }^{f} \quad$ Anne Brækhus ${ }^{c, g}, \mathrm{~h}$ \\ Bjørnar Hassel ${ }^{h, i} \quad$ Leiv Otto Watne ${ }^{a, j}$ \\ a Oslo Delirium Research Group, Department of Geriatric Medicine, Institute of Clinical \\ Medicine, University of Oslo, Oslo, Norway; ${ }^{b}$ Research Group for Lifespan Changes \\ in Brain and Cognition, Department of Psychology, University of Oslo, Oslo, Norway; \\ 'Department of Geriatric Medicine, Oslo University Hospital, Oslo, Norway; ${ }^{\mathrm{d}}$ Department \\ of Anesthesiology, Oslo University Hospital, Oslo, Norway; ${ }^{e}$ Department of Anesthesiology, \\ Diakonhjemmet Hospital, Oslo, Norway; ${ }^{f}$ Department of Orthopedic Surgery, Oslo \\ University Hospital, Oslo, Norway; ${ }^{9}$ Norwegian National Advisory Unit on Ageing and \\ Health, Vestfold Hospital Trust, Tønsberg, Norway; ${ }^{\text {h}}$ Department of Neurology, Oslo \\ University Hospital, Oslo, Norway; 'Norwegian Defense Research Establishment (FFI), Kjeller,

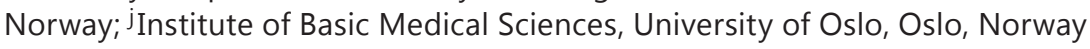

\section{Keywords}

Serum · Physiopathology · Hip fractures · Reference values · Delirium · Cerebrospinal fluid

\section{Abstract}

Aims: Phosphate is essential for neuronal activity. We aimed to investigate whether delirium is associated with altered phosphate concentrations in cerebrospinal fluid (CSF) and serum. Methods: Seventy-seven patients with hip fracture were assessed for delirium before and after acute surgery. Prefracture dementia was diagnosed by an expert panel. Phosphate was measured in CSF obtained immediately before spinal anesthesia $(n=77)$ and in serum $(n=$ 47). CSF from 23 cognitively healthy elderly patients undergoing spinal anesthesia was also analyzed. Results: Hip fracture patients with prevalent delirium had higher CSF phosphate concentrations than those without delirium (median $0.63 \mathrm{vs} .0 .55 \mathrm{mmol} / \mathrm{L}, p=0.001$ ). In analyses stratified on dementia status, this difference was only significant in patients with dementia. Serum phosphate was $\sim 1 \mathrm{mmol} / \mathrm{L}$; there was no association between serum phosphate concentration and delirium status. CSF phosphate did not correlate with serum levels. Conclusion: Patients with delirium superimposed on dementia have elevated phosphate levels. 


\section{Introduction}

Delirium is a transient neuropsychiatric syndrome that commonly occurs in the setting of an acute medical condition or trauma in elderly patients. The syndrome is characterized by its acute onset, fluctuating course, disturbance of consciousness and attention, cognitive dysfunction and perceptual disturbances. Delirium is associated with higher rates of institutionalization and death, and a greater risk of long-term cognitive decline [1-3]. The pathophysiology of delirium, however, is poorly understood [4].

Phosphate is essential for structural integrity of all cells and is an important compound in several cellular processes such as energy metabolism, intracellular signal transduction, and enzyme regulation. Hypophosphatemia in serum has been associated with neurological symptoms, e.g., delirium, hallucinations, irritability, and apathy [5]. Phosphate concentrations in cerebrospinal fluid (CSF) have never been assessed in delirium, but elevated CSF phosphate has been observed in neurological inflammatory diseases with altered mental status, e.g., meningitis and encephalitis [6].

The aim of this study was to assess possible associations between phosphate concentrations in serum and CSF and the occurrence of delirium in patients acutely operated for hip fracture.

\section{Materials and Methods}

\section{Participants}

The participants belonged to two cohorts. The first consisted of hip fracture patients included in the Oslo Orthogeriatrics Trial [7], recruited from September 2009 through January 2012. The second cohort consisted of cognitively healthy elderly patients undergoing elective orthopedic, gynecological, or urological surgery under spinal anesthesia, recruited from three different hospitals in Oslo from February 2012 to June 2013. In both cohorts, CSF was collected immediately before injection of the anesthetic agent.

\section{Cognitive Assessment}

In the hip fracture patients, delirium assessments were performed using the Confusion Assessment Method [8]. Patients were screened daily preoperatively and for 5 days postoperatively (all patients), or until discharge (patients with delirium). Delirium severity was assessed using the Memorial Delirium Assessment Scale [9]. The Informant Questionnaire on Cognitive Decline in the Elderly (IQCODE) [10] was filled out by a close relative or staff in nursing home or home nursing. Prefracture dementia was diagnosed by a geriatrician (T.B.W.) and a geriatric psychiatrist based on available information, e.g., IQCODE, case notes, and cognitive tests performed before and after the hip fracture. More detailed information on the delirium assessments and other cognitive measures have been published previously [11].

All cognitively healthy subjects underwent a thorough cognitive assessment prior to surgery. The assessment comprised the IQCODE [10], Clock Drawing Test [12], Trail Making Test [13], word list memory task from the Consortium to Establish a Registry for Alzheimer's Disease [14], Kendrick Object Learning Test [15], and verbal fluency [16] (Controlled Word Association Test, with the letters F, A and S, and Animal Naming).

\section{Selection Criteria}

Sample selection in the hip fracture cohort is the same as in a previously published article [17]. CSF was obtained from 143 patients with hip fracture. Our aim was to compare patients with delirium with patients free from any delirious symptoms, and thus we excluded patients 


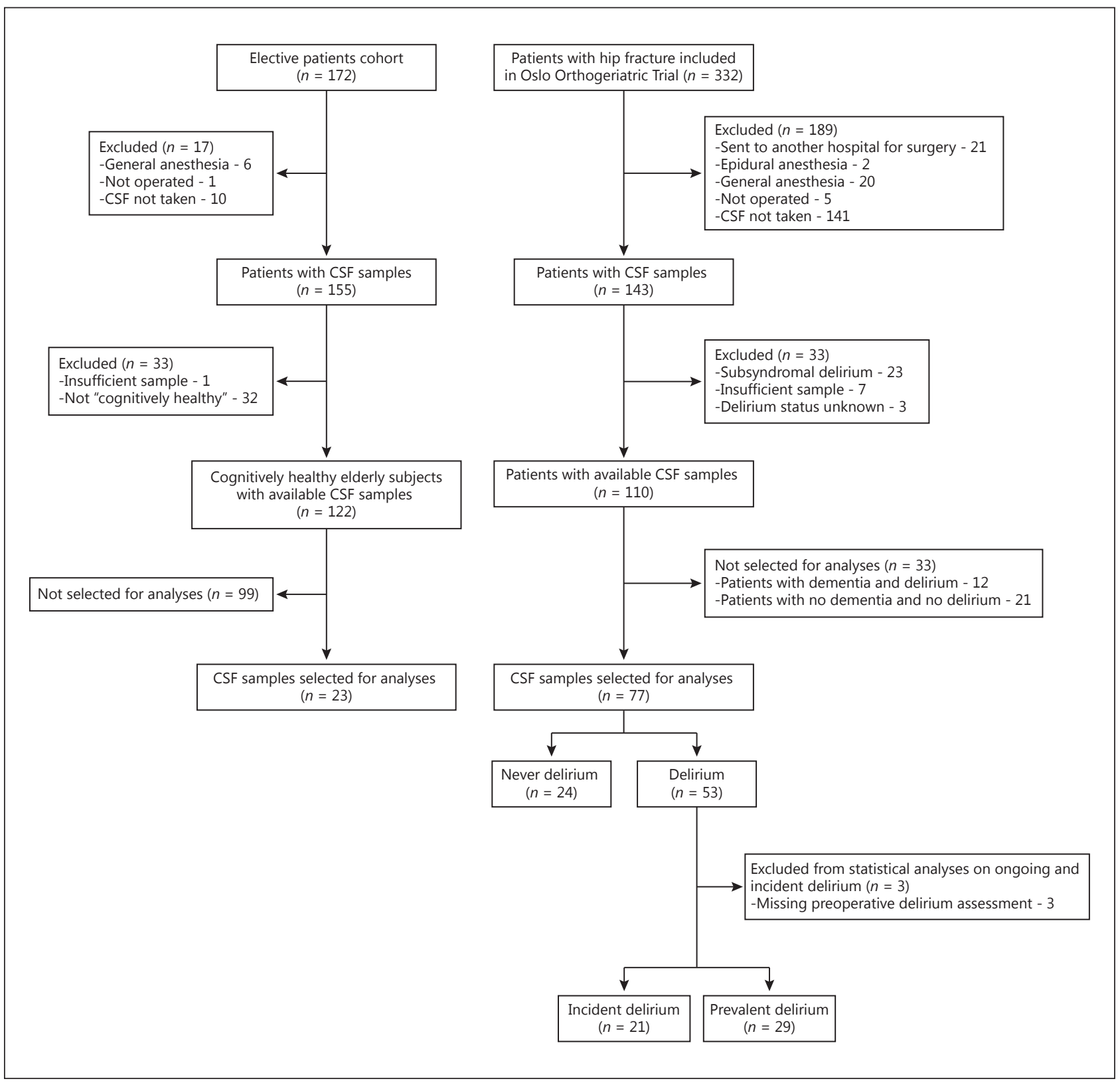

Fig. 1. Sample selection. Prevalent delirium, delirium at the time of CSF sampling; Incident delirium, developed delirium after CSF sampling; CSF, cerebrospinal fluid.

with subsyndromal delirium (defined as $\geq 1$ feature(s) using the Confusion Assessment Method, but never delirium). Patients with unknown delirium status were also excluded. We wanted all combinations of delirium and dementia status represented: (1) no delirium/no dementia, (2) delirium/no dementia, (3) no delirium/dementia, and (4) delirium/dementia.

There was a surplus of samples from groups 1 and 4, and we selected random samples from these two groups (Fig. 1). There were no significant differences in age, sex or IQCODE scores between patients included and patients excluded from group 1 and group 4, respectively. 
One hundred and seventy-one elective patients were cognitively tested, and only the cognitively healthy elderly subjects were included in this study. We defined "cognitively healthy" as no subjective cognitive impairment and $\leq 1$ abnormal test result on the abovementioned tests. Normal test results were defined as $\geq 4 / 5$ points on the Clock Drawing Test and $<2$ standard deviations below the mean for age, gender and educational level for all other tests. We selected a smaller group of patients for phosphate analyses (Fig. 1).

\section{Sample Handling and Assays}

CSF was collected in polypropylene tubes and centrifuged for $10 \mathrm{~min}$. Serum was collected preoperatively by venous puncture, left for clotting, and then centrifuged. Samples were aliquoted into polypropylene tubules and stored at $-80^{\circ} \mathrm{C}$. Phosphate levels were measured in CSF and serum samples by spectrophotometry (Vitros DT60 II spectrophotometer; OrthoClinical Diagnostics, Rochester, NY, USA). The analyses were done blindly with respect to clinical data.

\section{Statistical Analysis}

We analyzed continuous variables by the Kruskal-Wallis test and the Mann-Whitney U test due to a nonnormal distribution. Gender was analyzed by the Fisher exact test. Correlations were tested using Spearman correlations. In our primary analysis, we compared phosphate levels between hip fracture patients with and without delirium (not stratified on dementia status). In secondary analyses, we compared phosphate levels in CSF between three groups based on delirium status at the time of CSF sampling: hip fracture patients with prevalent delirium (delirium at the time of surgery), incident delirium (no delirium at the time of surgery, but developed delirium later), and never delirium (never any signs of delirium). These comparisons were also done stratified on prefracture cognitive function (dementia or no dementia). To adjust for potential confounding, we carried out two sets of linear regression analyses. One regression model was built for patients with dementia and one for patients without, both with delirium status (prevalent vs. incident vs. never delirium) as an independent variable and CSF phosphate as the dependent variable. IQCODE score, age, and gender were considered as potential confounding variables and introduced in the models separately and in different combinations.

\section{Ethical Considerations}

The studies were conducted in accordance with the Declaration of Helsinki. Written informed consent was obtained from the patients. If a patient with hip fracture was unable to give an informed consent, a presumed consent in combination with written assent from the closest relative was obtained. The studies were approved by the Regional Committee for Ethics in Medical Research in Norway (REK S-09169a and REK2011/2052).

\section{Results}

CSF samples from 77 patients with hip fracture and 23 cognitively healthy elderly subjects were analyzed. CSF and serum phosphate levels did not correlate in the whole patient sample, neither in the analyses taking into account dementia status nor in the analyses split according to delirium status. Thirty-eight of the patients with hip fracture had dementia. We found no difference in CSF phosphate levels between patients with and without dementia (median 0.60 vs. $0.56 \mathrm{mmol} / \mathrm{L}, p=0.09$ ). Fifty-three of the patients with hip fracture developed delirium. We found no significant difference in CSF phosphate levels between hip fracture patients with $(n=53)$ and without delirium ( $n=24)$ during their hospital stay (median $0.59 \mathrm{vs} .0 .52 \mathrm{mmol} / \mathrm{L}$, 


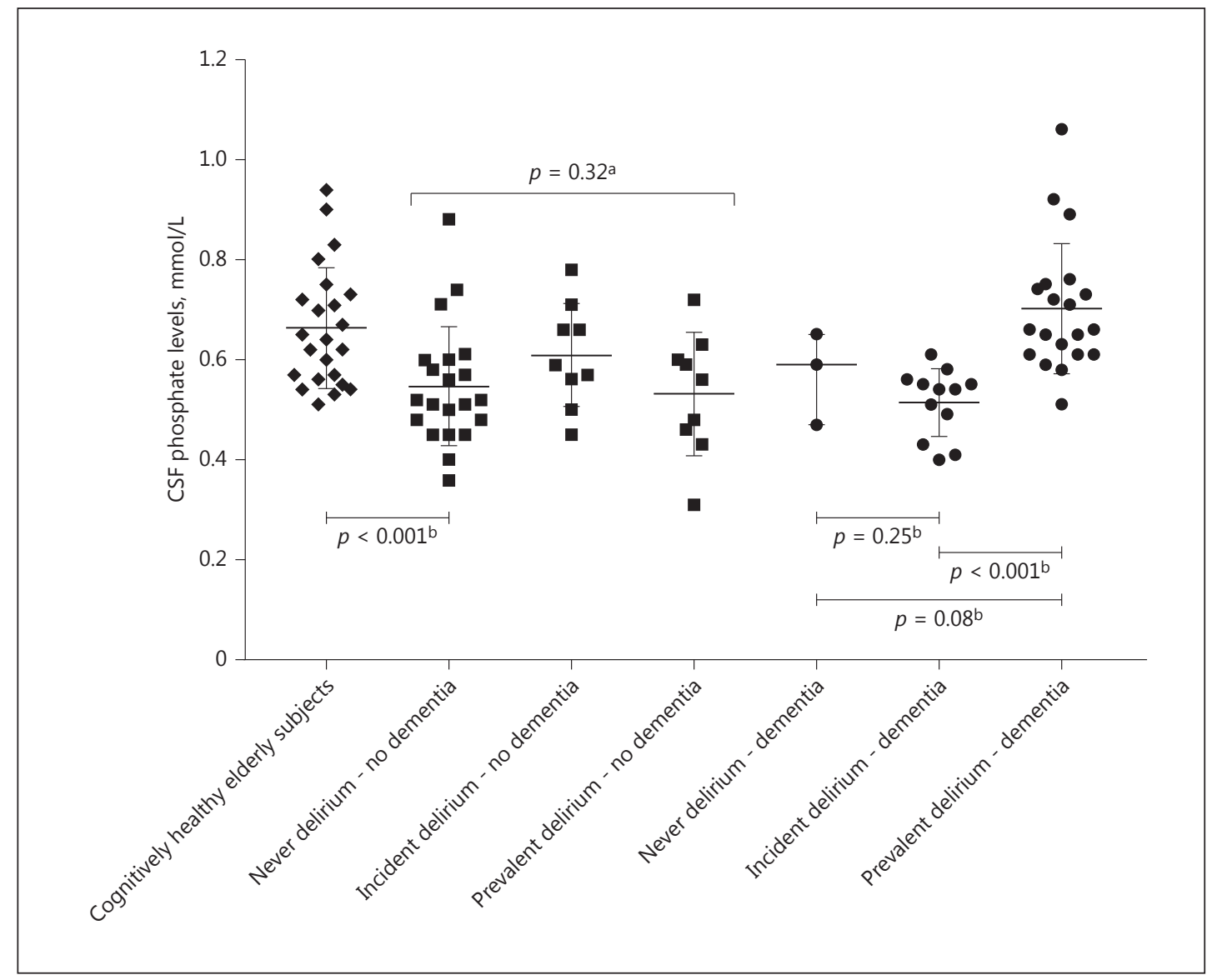

Fig. 2. Phosphate concentrations in CSF in cognitively healthy controls and in hip fracture patients with prevalent, incident or never delirium split according to prefracture dementia status. ${ }^{a}$ Kruskal-Wallis test (never delirium vs. incident delirium vs. prevalent delirium). ${ }^{b}$ Post hoc analyses (Mann-Whitney U test) in the dementia stratum indicated that statistically significant differences in CSF phosphate levels were: incident delirium versus prevalent delirium. Cognitively healthy elderly had significantly different CSF phosphate levels from hip fracture patients without delirium and dementia.

$p=0.08)$. Hip fracture patients with prevalent delirium $(n=29)$ at the time of CSF sampling had, however, significantly higher CSF phosphate levels than those without (incident delirium and never delirium combined, $n=45$; median 0.63 vs. $0.55 \mathrm{mmol} / \mathrm{L}, p=0.001$ ). In analyses stratified on dementia status, we found that this difference was present only in the dementia stratum. Patients with prevalent delirium and dementia had significantly higher levels than those with incident delirium (Fig. 2). In the no-dementia stratum, we found no significant differences in CSF phosphate levels related to delirium status (Fig. 2). The cognitively healthy elderly subjects had significantly higher CSF phosphate levels than hip fracture patients without delirium and dementia (Fig. 2). There were no statistically significant differences in serum phosphate levels between the delirium groups (Table 1).

Age, gender, and IQCODE scores were not significantly different between delirium status groups (Table 1). In linear regression models carried out separately for hip fracture patients with and without dementia, IQCODE score, age, and gender did not contribute to the explanation of the CSF phosphate levels; neither did introduction of these variables change the regression coefficients for the delirium grouping variables substantially (data not shown). 
Dementia

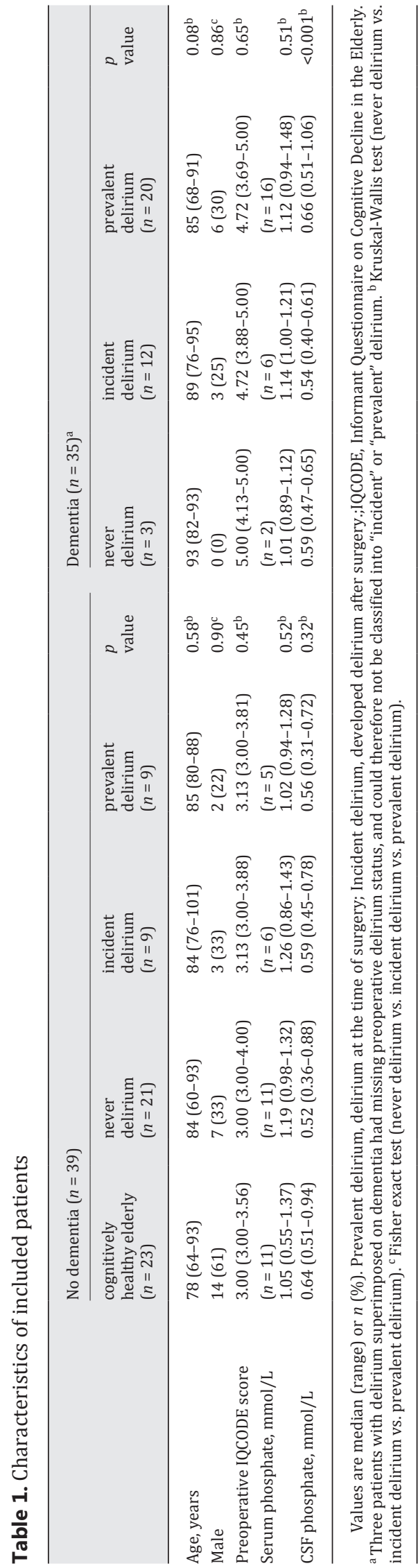


Memorial Delirium Assessment Scale maximum score during delirium was not correlated with CSF phosphate levels, neither in the whole sample $(r=0.004, p=0.98)$, nor in analyses split according to dementia status (dementia: $r=0.11, p=0.53$, no dementia: $r=$ $-0.39, p=0.13$ ).

\section{Discussion}

We report higher CSF phosphate levels in demented hip fracture patients with prevalent delirium than in patients without delirium. This is the first report of an association between CSF phosphate concentrations and delirium. There may be several sources of the higher CSF phosphate levels: first, damage to neurons or glial cells could cause an increase in extracellular phosphate that would lead to higher CSF levels, because the intracellular concentration of phosphate in the brain is higher than the extracellular concentration (1-1.5 mmol/L) [18]. Previous studies have shown evidence of cell damage in delirium, with elevated serum and CSF levels of the brain damage biomarker S100B during delirium $[19,20]$, although not consistently reported $[21,22]$. Second, increased neuronal activity has been shown to cause release of phosphate from neurons [23]. Hyperactive features of delirium, like restlessness, hyperalertness and psychotic symptoms, could represent such an increased neuronal activity, as suggested by a higher level of CSF lactate in patients with prevalent delirium [22]; lactate levels increase in the brain upon activation [24]. Leakiness of the blood-brain barrier, which theoretically could lead to influx of phosphate from the circulation, is an unlikely cause of the higher CSF level of phosphate in demented patients with delirium, because we did not find CSF phosphate to correlate with serum levels.

Further studies on cell damage and neuronal activity in delirium may shed light on why CSF phosphate levels increase in delirium. We did not find differences in CSF phosphate levels in hip fracture patients without dementia. This finding may suggest a difference in the pathogenesis of delirium in patients with and without dementia. It is likely that neurons with existing neurodegenerative pathology are more susceptible to harmful insults caused by delirium, possibly leading to brain cell damage, which may explain why a delirious episode accelerates the cognitive decline in demented patients $[2,3]$. Neuroinflammation, which is hypothesized to cause delirium, can create a neurotoxic response which is exacerbated in demented brains with primed microglia $[19,25]$. It is therefore possible that patients with delirium superimposed on dementia have a more widespread pathological process than patients without dementia, explaining why only these patients had higher CSF phosphate levels.

We found higher levels of CSF phosphate in cognitively healthy elderly subjects than in hip fracture patients without delirium and dementia. This underscores the importance of selecting valid control groups in delirium research. The cognitively healthy elderly subjects had CSF phosphate levels similar to those of demented hip fracture patients with delirium. The higher CSF phosphate levels in demented patients with delirium would have been lost if we had compared delirious hip fracture patients to cognitively healthy elderly subjects. However, a comparison limited to the group of demented hip fracture patients with and without delirium revealed a highly significant difference in CSF phosphate levels between the two groups.

The relatively large sample size is an important strength of our study. We were able to stratify analyses both according to dementia status and delirium status at the time of CSF sampling. This is important since dementia in itself may impact on CSF phosphate levels [26]. It was also possible to exclude patients with subsyndromal delirium, thus only including patients with a more accurate phenotyping. The fluctuating nature of delirium represents a limitation, as brief episodes of delirium may have been missed. Biomarker concentrations may also fluctuate, thus the cross-sectional design of our study is a weakness. 
Idland et al.: Cerebrospinal Fluid Phosphate and Delirium

\section{Conclusion}

CSF phosphate levels are higher in patients with delirium superimposed on dementia, suggesting a more widespread pathological process in delirium in these patients.

\section{Acknowledgements}

The study was mainly funded by the Research Council of Norway through the program "Improving mental health of older people through multidisciplinary efforts" (grant No. 187980/H10). Further, we have received funding from Oslo University Hospital, The Sophies Minde Foundation, The Norwegian Association for Public Health, Civitan's Research Foundation, South-Eastern Norway Regional Health Authority, and the Medical Student Research Program at the University of Oslo. The funders had no role in the design, methods, subject recruitment, data collections, analysis, or preparation of paper.

The authors would like to thank Knut Engedal, MD, PhD (University of Oslo and Vestfold Hospital Trust) for his contribution to the dementia consensus diagnoses, Anette Hylen Ranhoff, MD, PhD (Diakonhjemmet Hospital and Kavli Research Center for Geriatrics and Dementia) for general support, and Elisabeth Fragaat (Oslo University Hospital), Tone Fredriksen (Oslo University Hospital), Camilla Marie Andersen (Oslo University Hospital), Julie Ask Ottesen (Oslo University Hospital), Linda Feldt (Oslo University Hospital), Arne Myklebust, MD (Oslo University Hospital), and Dagfinn Tore Kollerøs, MD (Oslo University Hospital) for collection of data. The authors would also like to thank the study participants, and acknowledge the contributions of the Department of Gynecology, the Department of Urology, the Department of Orthopedic Surgery, Department of Geriatric Medicine and the Department of Anesthesiology at Oslo University Hospital, the Department of Orthopedic Surgery and the Department of Anesthesiology at Diakonhjemmet Hospital in Oslo, Norway.

\section{Disclosure Statement}

Dr. Wyller has given lectures on delirium for Pfizer, Roche, AstraZeneca and Nycomed. Dr. Watne has given a lecture on delirium for Lilly. Dr. Frihagen has received financial support for education from Eli Lilly Norway, Takeda Nycomed and Amgen Norway. Frihagen has given lectures and received honoraria from Eli Lilly Norway and Amgen Norway. The other authors report no conflicts of interest.

\section{References}

$>1$ Witlox J, Eurelings LS, de Jonghe JF, Kalisvaart KJ, Eikelenboom P, van Gool WA: Delirium in elderly patients and the risk of postdischarge mortality, institutionalization, and dementia: a meta-analysis. JAMA 2010;304: 443-451.

2 Davis DH, Muniz Terrera G, Keage H, Rahkonen T, Oinas M, Matthews FE, Cunningham C, Polvikoski T, Sulkava $\mathrm{R}$, MacLullich AM, Brayne C: Delirium is a strong risk factor for dementia in the oldest-old: a population-based cohort study. Brain 2012;135:2809-2816.

-3 Davis DH, Muniz-Terrera G, Keage HA, Stephan BC, Fleming J, Ince PG, Matthews FE, Cunningham C, Ely EW, MacLullich AM, Brayne C: Association of delirium with cognitive decline in late life: a neuropathologic study of 3 population-based cohort studies. JAMA Psychiatry 2017;74:244-251.

4 Fong TG, Davis D, Growdon ME, Albuquerque A, Inouye SK: The interface between delirium and dementia in elderly adults. Lancet Neurol 2015;14:823-832.

-5 Subramanian R, Khardori R: Severe hypophosphatemia. Pathophysiologic implications, clinical presentations, and treatment. Medicine (Baltimore) 2000;79:1-8. 
-6 Heipertz R, Eickhoff K, Karstens KH: Magnesium and inorganic phosphate content in CSF related to bloodbrain barrier function in neurological disease. J Neurol Sci 1979;40:87-95.

7 Watne LO, Torbergsen AC, Conroy S, Engedal K, Frihagen F, Hjorthaug GA, Juliebo V, Raeder J, Saltvedt I, Skovlund E, Wyller TB: The effect of a pre- and postoperative orthogeriatric service on cognitive function in patients with hip fracture: randomized controlled trial (Oslo Orthogeriatric Trial). BMC Med 2014;12:63.

-8 Inouye SK, van Dyck CH, Alessi CA, Balkin S, Siegal AP, Horwitz RI: Clarifying confusion: the confusion assessment method. A new method for detection of delirium. Ann Intern Med 1990;113:941-948.

-9 Breitbart W, Rosenfeld B, Roth A, Smith MJ, Cohen K, Passik S: The Memorial Delirium Assessment Scale. J Pain Symptom Manage 1997;13:128-137.

10 Jorm AF: A short form of the Informant Questionnaire on Cognitive Decline in the Elderly (IQCODE): development and cross-validation. Psychol Med 1994;24:145-153.

11 Wyller TB, Watne LO, Torbergsen A, Engedal K, Frihagen F, Juliebo V, Saltvedt I, Skovlund E, Raeder J, Conroy S: The effect of a pre- and post-operative orthogeriatric service on cognitive function in patients with hip fracture. The protocol of the Oslo Orthogeriatrics Trial. BMC Geriatr 2012;12:36.

12 Shulman KI: Clock-drawing: is it the ideal cognitive screening test? Int J Geriatr Psychiatry 2000;15:548-561.

13 Reitan RM: The relation of the Trail Making Test to organic brain damage. J Consult Psychol 1955; 19:393-394.

14 Morris JC, Heyman A, Mohs RC, Hughes JP, van Belle G, Fillenbaum G, Mellits ED, Clark C: The Consortium to Establish a Registry for Alzheimer's Disease (CERAD). Part I. Clinical and neuropsychological assessment of Alzheimer's disease. Neurology 1989;39:1159-1165.

15 Kendrick DC, Gibson AJ, Moyes ICA: The revised Kendrick battery: clinical studies. Br J Soc Clin Psychol 1979; 18:329-340.

16 Spreen 0, Strauss E: A Compendium of Neuropsychological Tests: Administration, Norms, and Commentary. Oxford, Oxford University Press, 1991.

17 Watne LO, Idland AV, Fekkes D, Raeder J, Frihagen F, Ranhoff AH, Chaudhry FA, Engedal K, Wyller TB, Hassel B: Increased CSF levels of aromatic amino acids in hip fracture patients with delirium suggests higher monoaminergic activity. BMC Geriatr 2016;16:149.

18 Barbiroli B, Montagna P, Martinelli P, Lodi R, Iotti S, Cortelli P, Funicello R, Zaniol P: Defective brain energy metabolism shown by in vivo 31P MR spectroscopy in 28 patients with mitochondrial cytopathies. J Cereb Blood Flow Metab 1993;13:469-474.

19 van Munster BC, Korse CM, de Rooij SE, Bonfrer JM, Zwinderman AH, Korevaar JC: Markers of cerebral damage during delirium in elderly patients with hip fracture. BMC Neurol 2009;9:21.

$\longrightarrow 20$ Hall RJ, Ferguson KJ, Andrews M, Green AJ, White TO, Armstrong IR, Maclullich AM: Delirium and cerebrospinal fluid S100B in hip fracture patients: a preliminary study. Am J Geriatr Psychiatry 2013;21:1239-1243.

-21 Beishuizen SJ, Scholtens RM, Vellekoop AE, Vrouenraets BC, Westhoff D, van de Beek D, de Rooij SE, van Munster BC: Timing is critical in determining the association between delirium and S100 calcium-binding protein B. J Am Geriatr Soc 2015;63:2212-2214.

$\checkmark 22$ Caplan GA, Kvelde T, Lai C, Yap SL, Lin C, Hill MA: Cerebrospinal fluid in long-lasting delirium compared with Alzheimer's dementia. J Gerontol A Biol Sci Med Sci 2010;65:1130-1136.

23 Ritchie JM, Straub RW: Increase in efflux of inorganic phosphate during electrical activity in small nonmyelinated nerve fibres. J Physiol 1978;274:539-548.

24 Dienel GA, Cruz NF: Aerobic glycolysis during brain activation: adrenergic regulation and influence of norepinephrine on astrocytic metabolism. J Neurochem 2016;138:14-52.

25 van Gool WA, van de Beek D, Eikelenboom P: Systemic infection and delirium: when cytokines and acetylcholine collide. Lancet 2010;375:773-775.

26 Subhash MN, Padmashree TS, Srinivas KN, Subbakrishna DK, Shankar SK: Calcium and phosphorus levels in serum and CSF in dementia. Neurobiol Aging 1991;12:267-269. 